\title{
Metalurgia e materiais \\ Desenvolvimento de uma metodologia para medição do atrito entre tira e mandril da bobinadeira de laminador Sendzimir
}

\section{Development of a methodology for measuring the friction between the strip and coiler mandrel of Sendzimir mill}

\begin{abstract}
Antônio Carlos Ataíde
Engenheiro Mecânico, Aperam South America antoniocarlos.ataide@yahoo.com.br
\end{abstract}

Fernando Cláudio de Oliveira

Engenheiro Eletricista, Aperam South America fernando.claudio@arcelormittal.com.br

Antônio Eustáquio de Melo Pertence Professor Associado do Departamento de Engenharia Mecânica - UFMG pertence@demec.ufmg.br

Paulo Roberto Cetlin

Professor Titular do Departamento de

Engenharia Mecânica - UFMG

pcetlin@demet.ufmg.br

\section{Resumo}

Os laminadores a frio da Aperam South America são equipados com mandris que têm a função de sustentar o peso da bobina e de transmitir torque proveniente do acionamento. Os mandris são dotados de mecanismos chamados mordente, cuja função é prender a ponta da tira no início do processo de enrolamento e, assim, permitir a aplicação da tração de bobinamento. A prática atual de bobinamento considera que a aplicação da tração somente pode ser iniciada após, pelo menos, 1,5 voltas de tira no mandril, o que gera perda de rendimento físico nos laminadores. Foi desenvolvida uma metodologia para medição do atrito entre a tira e a superfície do mandril, numa aproximação das condições industriais, tendo em vista estudos posteriores para avaliação da capacidade dos mordentes dos mandris, a fim de se aplicar a tração com menos de uma volta de tira enrolada no mandril. Desenvolveu-se um modelo físico que foi construído próximo da escala real, o qual foi submetido a ensaios experimentais envolvendo segmentos dos mandris de dois laminadores. Os resultados do atrito médio na interface tira e mandril estão coerentes com os usados na literatura.

Palavras-chave: Laminador, mandril, mordente.

\begin{abstract}
The cold rolling mills of Aperam South America are equipped with mandrels which have the function of sustaining the coil load and torque transmission from the motor. The mandrels are equipped with mechanisms called grippers, whose function is to grip the tail of the strip at the beginning of the wrapping process and thus permit the winding tension application. The current winding practice considers that tension application can only be started after the wrapping up of at least 1.5 turns of the strip onto the mandrel, which generates a decline in the physical performance of the rolling mills. A methodology was developed to measure the friction between the strip and the surface of the mandrel, under approximate industrial conditions in order to further studies for evaluating madrel gripper capacity under an applied tension of less than one turn of the strip around the mandrel. A near-scale physical model was built, which was subjected to experimental tests involving segments of mandrels from two mills. The results of the average friction at the interface strip and mandrel are consistent with those found in literature.
\end{abstract}

Keywords: Mill, mandrel, gripper. 


\section{Introdução}

Os laminadores a frio tipo Sendzimir da Aperam South America são equipados com bobinadeiras, que têm a função de enrolar a bobina sobre um mandril expansível, na tração adequada ao processo. Os mandris são dotados de um mecanismo chamado mordente, que é montado em um dos quatro segmentos, cuja função é prender a ponta da tira, a fim de se iniciar o enrolamento e de se permitir a aplicação da tração.

A prática atual de bobinamento considera que a aplicação da tração somente pode ser iniciada após a tira ter completado pelo menos 1,5 volta no mandril. Dessa forma, a tração pode ser

\section{Materiais e métodos}

$\mathrm{O}$ atrito, na interface entre a tira e o mandril, tem considerável importância nas operações de bobinamento de chapas metálicas. $\mathrm{O}$ atrito ocorre na região do mordente onde a tira é presa e na região conformada ao longo do mandril, logo tem uma contribuição importante para a força total de bobinamento.

Segundo Sefton (2000), existem, basicamente, duas formas para medição do atrito, por medição direta ou indireta. $\mathrm{Na}$ medição indireta, o atrito pode ser obtido, por exemplo, medindo a força tangencial e a força normal e, através de equações matemáticas, determina-se o atrito médio para uma determinada área de contato. Na medição direta, utilizamse sensores especiais, com os quais é possível determinar a distribuição do atrito dentro de uma área de contato aparente. Nesse trabalho, utilizou-se uma metodologia para determinação do atrito médio entre a tira e o mandril através de medição indireta.

Para a execução dos testes, foi desenvolvido um dispositivo de forma a obter uma cópia física dos mandris dos

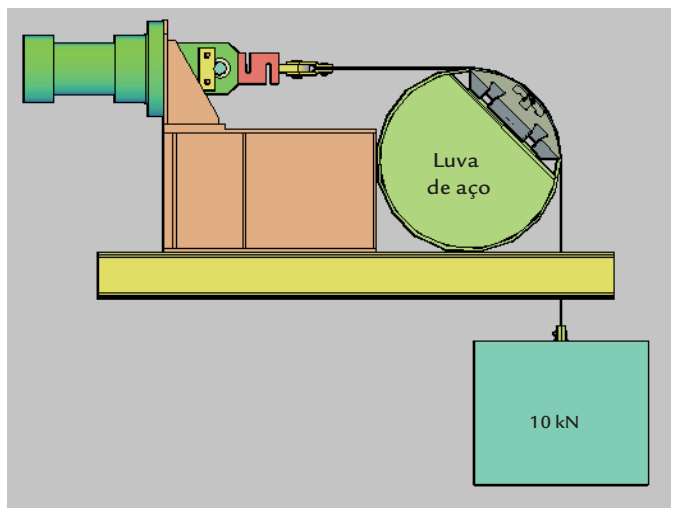

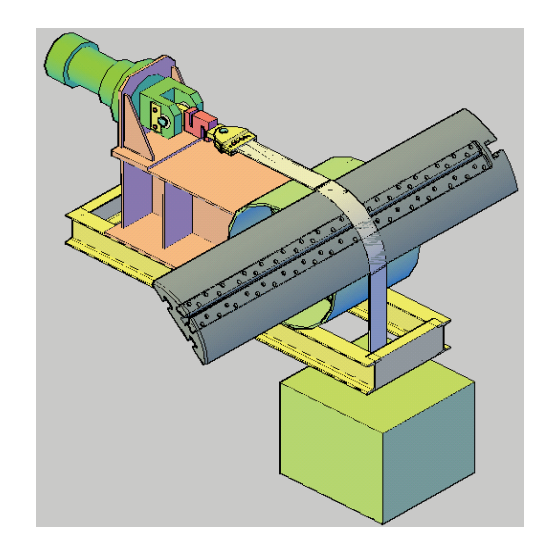

laminadores a frio da Aperam South America, próximos da escala real, onde se utilizam os próprios segmentos dos mandris, para a realização dos experimentos. Tal dispositivo é composto pelos seguintes componentes:

- Componentes estruturais para sustentar o segmento e o cilindro hidráulico.

- Garra para conectar a tira à célula de carga.

- Célula de carga de tração de capacidade de $50 \mathrm{kN}$ para medir a tração na tira.

- Cilindro hidráulico com diâmetro de camisa de $140 \mathrm{~mm}$, diâmetro da haste de $85 \mathrm{~mm}$ e curso de $85 \mathrm{~mm}$ para tracionar a tira.

- Indicador de força para aquisição dos dados medidos pela célula de carga.

- Uma unidade hidráulica para alimentação do cilindro hidráulico de tração.

- Sistema de aquisição capaz de registrar e analisar os dados coletados.

A Figura 1 apresenta o arranjo do dispositivo para medição do atrito. tensão das pontas descartadas como heavy gange depende do equipamento e é composta de um comprimento fixo que se refere à distância do centro da cadeira de laminação até o eixo do mandril, e de um comprimento variável, que depende do quanto a tira está enrolada no mandril. Desta forma, tem-se uma perda fixa e uma perda variável.

Nesse trabalho, desenvolveu-se uma metodologia para medição do atrito entre a tira e o mandril, a fim de se fornecerem subsídios para estudos posteriores de avaliação da tração de bobinamento com o menor arco de contato possível, visando à redução da perda variável.
A medição do atrito pretende simular o deslizamento da tira sobre os segmentos dos mandris. O método escolhido foi baseado em American Society for Testing and Materials - ASTM G143 (2009), que define um sistema similar ao dispositivo apresentado na Figura 1.

Os segmentos dos mandris do LB1 (Laminador de bobinas número 1) e LB4 (Laminador de bobinas número 4) foram fixados na luva de aço e posicionados conforme mostra a Figura 1, fazendo um ângulo de $45^{\circ}$ com a horizontal, de forma que a tira tenha contato apenas com o segmento.

Foram utilizadas tiras do aço AISI

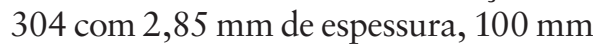
de largura e $1400 \mathrm{~mm}$ de comprimento, cortadas na mesma direção de laminação da chapa.

A Tabela 1 apresenta a composição química do material e, na Tabela 2, encontram-se as propriedades mecânicas.

Uma extremidade da tira foi fixada à célula de carga conforme mostrado na Figura 2 e a outra foi interligada a um peso-padrão de $10 \mathrm{kN}$.

Figura 1

Arranjo do dispositivo para medição de atrito. 
Tabela 1

Composição química do aço inoxidável austenítico - AISI 304.

Tabela 2

Propriedades mecânicas do aço inoxidável austenítico - AISI 304.

Figura 2

Detalhe da fixação da tira à célula de carga.

Tendo em vista assegurar a confiabilidade da medição, a célula de carga foi calibrada utilizando-se pesos-padrão. Foram içados três pesos conhecidos com a célula de carga na posição vertical e foi ajustado o indicador de força.

Não há uma distância mínima de deslizamento da tira sobre a superfície cilíndrica, que, neste caso, é o próprio segmento do mandril. Se o objetivo for apenas o atrito estático, basta sair da velocidade zero, deslocar poucos milímetros e, se for o atrito cinético, a distância deve ser suficiente para que a força aplicada pelo atuador permaneça constante (ASTM G143, 2009).

Tabela 3

Valores médios de rugosidades medidas nos segmentos e tiras metálicas.

Conforme modelo desenvolvido

onde $F 1$ é a força-peso, F2 é força medida pela célula de carga, $F b$ é a força de flexão da tira e $\theta$ é o arco de contato for-

onde, $\sigma_{y}$ é o limite de escoamento do material, $t_{0}^{y}$ é a espessura da tira, $L$ é a largura da tira e $R$ é o raio da luva de aço.

\begin{tabular}{c|c|c|c|c|c|c|c}
\hline \multicolumn{1}{c}{ Composição Química (\% peso) } \\
\hline $\mathbf{C}$ & $\mathbf{M n}$ & $\mathbf{P}$ & $\mathbf{S i}$ & $\mathbf{N i}$ & $\mathbf{C r}$ & $\mathbf{S}$ & Co \\
\hline 0,04 & 1,20 & 0,03 & 0,37 & 8,01 & 18,23 & 0,03 & 0,16 \\
\hline
\end{tabular}

Fonte - Laboratório de análise química da Aperam South America.

\begin{tabular}{c|c|c|c}
\hline $\begin{array}{c}\text { Limite de Resistência } \\
(\mathrm{MPa})\end{array}$ & $\begin{array}{c}\text { Limite de Escoamento } 0,2 \% \\
(\mathrm{MPa})\end{array}$ & $\begin{array}{c}\text { Alongamento } \\
\text { Percentual (\%) }\end{array}$ & Dureza (HRB) \\
\hline 709,16 & 363,47 & 50,6 & 89 \\
\hline
\end{tabular}

Fonte - Laboratório de ensaios mecânicos da Aperam South America.

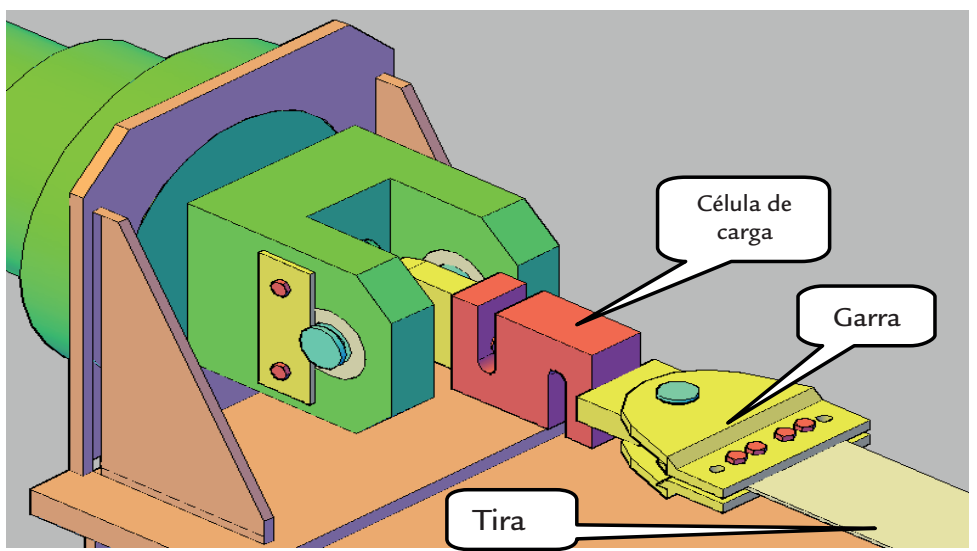

Os testes foram executados nas condições a seco, o mandril foi lubrificado com óleo mineral, utilizado para refrigeração da chapa, durante o processo de laminação, e foi lubrificado com a mistura do mesmo óleo mineral com graxa a base de lítio e dissulfeto de molibdênio, graxa que é utilizada na lubrificação dos mandris. O óleo mineral utilizado possui viscosidade de $8 \mathrm{cSt}$ a $40^{\circ} \mathrm{C}$ e densidade de $860 \mathrm{~kg} / \mathrm{m}^{3}$. Foi realizada limpeza no segmento e nas tiras de aço com Thinner 3500 para remoção da oleosidade e dos resíduos.

Foram medidas as rugosidades dos segmentos e das tiras de aço AISI
304, cujos valores estão registrados na Tabela 3 .

Uma extremidade da tira foi interligada ao dispositivo e a outra ao peso tensionador, de forma alinhada seguindo as marcações feitas no segmento.

Foi ajustada a velocidade média do cilindro de tração em $7 \mathrm{~mm} / \mathrm{s}$, feito um pequeno deslocamento, apenas o suficiente para retirar o peso do solo e aguardado o tempo de $60 \mathrm{~s}$ para estabilização da carga. Na sequência, foi realizado o deslocamento referente ao curso restante do cilindro hidráulico de $70 \mathrm{~mm}$. Nessa operação, foram registrados e gravados os sinais de força sobre a tira.

\begin{tabular}{c|c|c}
\hline Segmento do LB1 & Segmento do LB4 & Tiras metálicas \\
\hline $2,1 \mathrm{R}_{\mathrm{a}}$ & $1,6 \mathrm{R}_{\mathrm{a}}$ & $3,0 \mathrm{R}_{\mathrm{a}}$ \\
\hline
\end{tabular}

por Saha et al. (1996), o atrito médio $(\mu)$

$$
\mu=2 \frac{(F 2-F 1-F b)}{\theta(F 1+F 2)}
$$

mado entre a tira e o cilindro.

A diferença entre a força medida $(F 2)$ e a força-peso $(F 1)$ é devida ao atrito

$$
F b=\frac{\sigma_{\mathrm{y}} \cdot t_{0}^{2} \cdot L}{2 \cdot R}
$$

Para cada teste replicado, foi utilizada uma nova tira metálica e realizados os mesmos procedimentos de limpeza. pode ser calculado através da EQ. (1).

$(\mu)$ e à força requerida para flexão da tira (Fb), que, segundo Swift (1948), pode ser obtida através da EQ. (2).

Foram realizados três testes para cada condição: a seco, lubrificado com óleo e lubrificado com óleo e graxa. 
A Figura 3 apresenta o conjunto montado que foi utilizado para avaliação

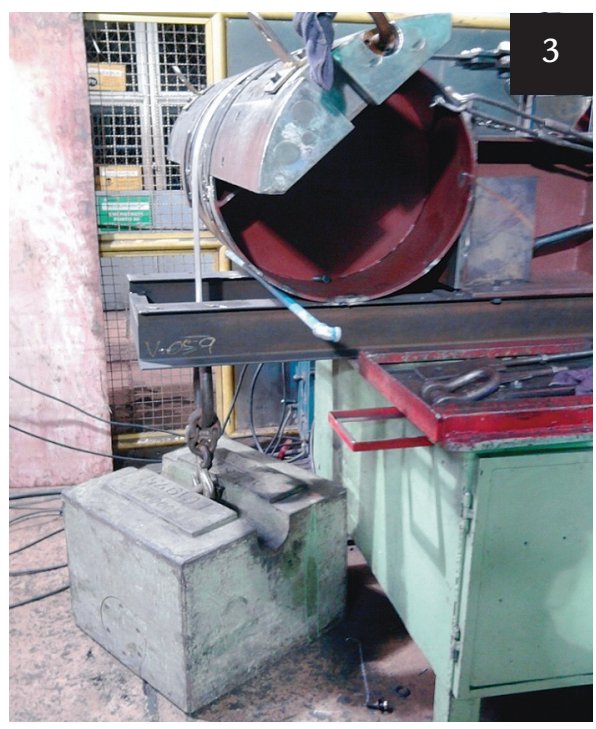

\section{Resultados e discussão}

O método de medição indireta utilizado apresentou resultados consistentes e boa repetibilidade. A Figura 5 apresenta os resultados de três testes realizados na condição a seco, com deslizamento da tira sobre o segmento do mandril do do atrito da tira com o segmento, onde se destaca o peso utilizado para tensiona-

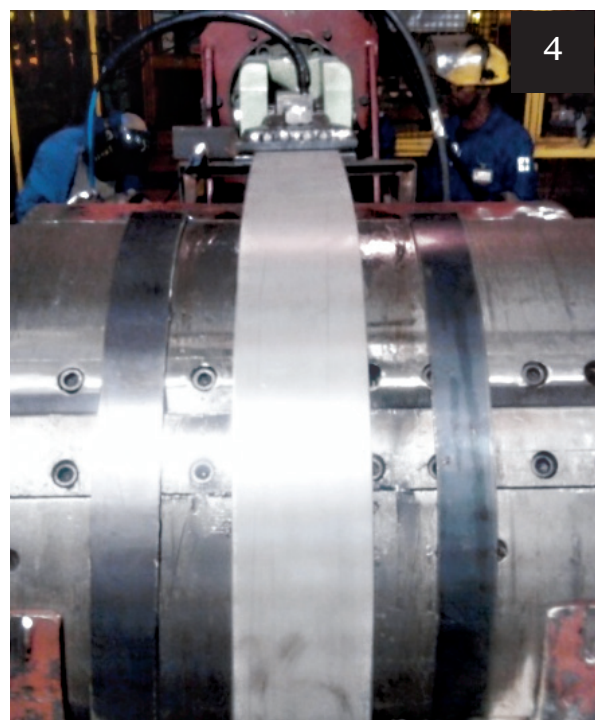

mento da tira. A Figura 4 mostra o detalhe da tira conformada sobre o segmento.

Figura 3

Conjunto montado

para avaliação do atrito.

Figura 4

Detalhe da tira conformada sobre o segmento.

LB1. Observa-se boa similaridade dos resultados.

Realizaram-se novos testes para três condições de lubrificação: a seco, lubrificado com óleo mineral utilizado no processo de laminação, e lubrificado com
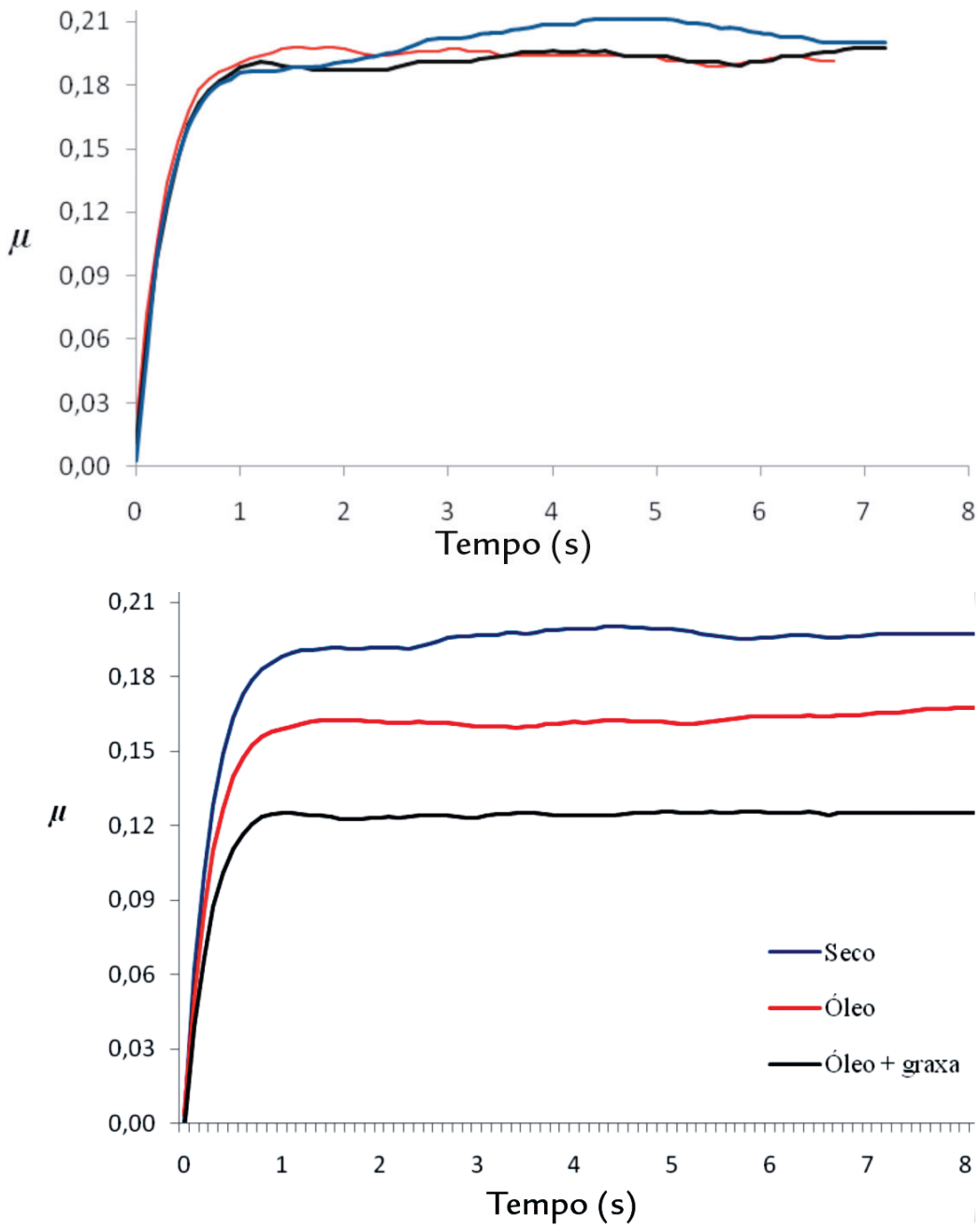

a mistura de óleo e graxa, esta utilizada na lubrificação dos mandris. Os testes apresentaram diferentes resultados em função da condição de lubrificação. A Figura 6 apresenta os valores de atrito para três condições.
Figura 5

Repetibilidade na medição do atrito a seco entre tira e segmento do LB1.
Figura 6

Influência da lubrificação no atrito tira/segmento do LB1. 
Observa-se na Figura 6, uma diferença significativa do atrito na condição de lubrificação com óleo e graxa, situação muito comum no processo industrial, com a condição a seco

De forma idêntica ao caso anterior, realizou-se a medição do atrito entre a tira e o segmento do mandril do LB4. Os testes foram executados para três condições de lubrificação: a seco, mandril lu-

Figura 7

Influência da lubrificação no atrito tira/segmento do LB4.

\section{Conclusão}

No presente trabalho, foi apresentada uma metodologia para avaliar o atrito na interface entre a tira e o mandril de bobinadeira de laminador Sendzimir. A aplicação da metodologia, para a determinação do atrito médio, em dois casos experimentais, apresentou resultados satisfatórios, uma vez que os resultados obtidos estão coerentes com os usados na

\section{Agradecimentos}

Os autores agradecem à Aperam South America, em nome dos gerentes,

\section{Referências bibliográficas}

brificado com óleo mineral, utilizado no processo de laminação, e mandril lubrificado com a mistura de óleo e graxa, esta utilizada na lubrificação dos mandris. A Figura 7 apresenta os valores de atrito para três condições.

Observa-se na Figura 7, a diferença dos valores do atrito em função da condição de lubrificação. Fazendo uma comparação com os valores obtidos no

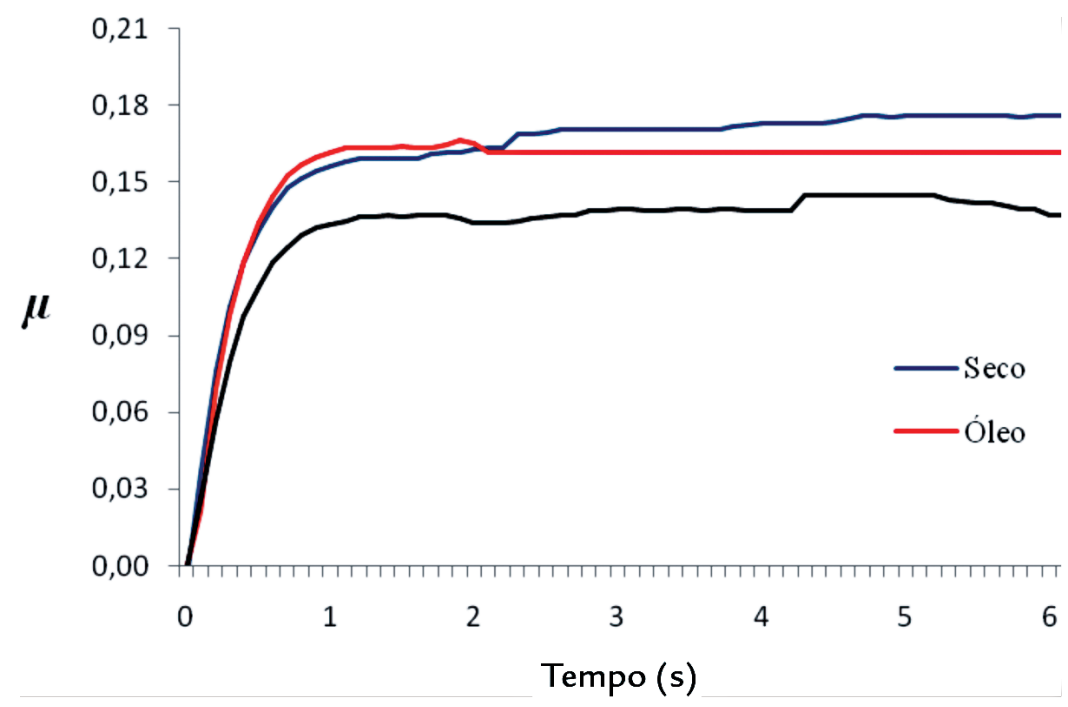

literatura.

As condições de lubrificação permitiram reproduzir os vários tipos de contato presentes na situação real que envolve o bobinamento de tiras.

Tendo em vista que a qualidade das superfícies dos segmentos interferiu nos resultados do atrito, recomenda-se a aplicação da metodologia para outros mate- experimento realizado com o segmento do mandril do LB1, observa-se uma pequena diferença dos valores de atrito para a condição de lubrificação com óleo + graxa. Observa-se, ainda, que o atrito na condição a seco referente a este caso apresentou valores menores que o caso anterior. Acredita-se que essa diferença seja devida à menor rugosidade do segmento do LB4.
Fernando Claudio de Oliveira e Vicente Dambroz, pela cessão das imagens e base riais da família dos aços inoxidáveis, variando a rugosidade das tiras metálicas.

Essa metodologia pode ser melhorada com a utilização de um servo-sistema em malha fechada equipado com servo-válvula, a fim de se melhorar o controle de força e da velocidade de deslizamento da tira sobre a superfície do segmento. de dados e pela fabricação dos equipamentos.
ASTM G 143. Standard Test Method for Measurement of Web/Roller Friction Characteristics. American society for Testing and Materials. 2009.

SAHA, P. K., WILSON, W. R. D., TIMSIT, R. S. Influence of surface topography on the frictional characteristics of 3104 aluminum alloy sheet. Wear, v.197, p. 123$129,1996$.

SEFTON, H. A friction sensor for a sheet metal forming simulator. Ontário: Queen's University Kingston, 2000. (Dissertação de mestrado).

SWIFT, L.D. Plastic bending under tension. Engineering, v.166, p. 333-359, 1948.

T. SENDZIMIR, Inc. Reverese pyramid winder mandrels. disponível em: http:/ sendzimir.com. Acessado em 15 de Set. 2010.

TURLEY, J.W. Sendzimir controlled collapse winder. Iron and Steel Engineer, v. 51, n. 42, 1974. 